\title{
No Scientific Clinical Evidence for long term Safety of Newly Evolved COVID_19 Vaccines: Review and Meta-analysis.
}

Editor-in Chief Article

Yasser Helmy Ali, MD

*Corresponding Author:

Yasser Helmy Ali

dryasserhelmy@azhar.edu.eg

dryasserhelmy@gmail.com

Editor article submitted and published on December24, 2020

Copyright 2020 The Authors published by Al-Azhar University, Faculty of Medicine, Cairo, Egypt. All rights reserved. This an openaccess article distributed under the legal terms, where it is permissible to download and share the work provided it is properly cited. The work cannot be changed in anyway or used commercially.

DOI: 10.21608/aimj.2020.55247.1382

${ }^{1}$ AIMJ Editor-in Chief, Chief of

scientific research unit,

Professor of Plastic surgery,

Faculty of Medicine, Al-Azhar

University, Cairo, Egypt.

Authorship: Author has a total contribution to the article.

Disclosure: Author has no financial interest to declare in relation to the content of this article.

\begin{abstract}
Background: Since the Corona pandemic began a year ago, the world has held its breath. Everyone is looking for hope to end this epidemic that has killed hundreds of thousands and sickened millions around the world. This will only be achieved by acquiring community immunity by either infecting the largest number of the planet's population or producing an effective and safe vaccine. In the past few days, companies of Vaccine developers and governments around the world announced, in media statements, that the vaccine production experiments have been successful and that its effectiveness has exceeded $90 \%$. So governmental institutions, led by the US Food and Drug Administration, have provided an emergency permit to administer the vaccine to humans.
\end{abstract}

Aim of the work: Here the question arises, how safe are those vaccines? especially since clinical trials may haven't been fulfilled as required because of the emergency situation. These vaccines will be given to billions of people with different genetic predispositions and different age groups and disease states, which means a differentiated immune response. ${ }^{2}$ Perhaps this immune response could be dangerous or unexpected, especially in some groups of people, such as the elderly and children, as well as workers in the health sectors and many others, who are mainly targeted by the vaccine. Then what are the immunological results that may appear after a year or two or after many years. Also, data deficiency burdens the regulatory process by the FDA and other governmental health authorities.

Methods: This article searches the scientific answers based on metaanalysis for published articles in Scopus and Clarivate data bases.

In conclusion, there is no scientific clinical evidence for long term safety of newly evolved COVID_19 Vaccines.

Keywords: COVID_19; Vaccines; Safety; long Term; Evidenc

\section{Safety Evidence}

In March 2020 an article written by Shibo Jiang $^{3}$ in Nature titled: Don't rush to deploy COVID19 vaccines and drugs without sufficient safety guarantees. Author has recommended proper and strict guidelines of clinical trials to avoid any possible potential vaccine side effects. He stated literally that; regulators must continue to require that vaccine developers check for potentially harmful responses in animal studies.

In this stage Shibo was concerned and has worried about the administration of Vaccine before full efficacy and safety profile approved in both preclinical and clinical trials. Scientific community understands the ultimate needs for Vaccinations because of the COVID_19 emergency situation in all over the world but no Scientist could disagree with Shibo in that: testing vaccines in harry without proper assessment of the safety risks profile could bring unwarranted setbacks either in the current pandemic, or in the future. (did developers measure twice, cut once as old say or cut with one or nonmeasure! $)^{3}$. The main question is could scientists have the data that enable them to analyze the safety of these vaccines in meta-analysis design according to what are published in scientific articles around the world, and not according to press releases issued from here or there.

Author of this article has searched in Scopus and Clarivate data bases to find outcome follow up of clinical trials for COVID_19 evolved vaccines, the search result about both; Vaccine design and safety was only 81 articles in Scopus and 42 in Clarivate till December $1^{\text {st }}, 2020$. Total articles are 123 out of them 29 were excluded as they were irrelevant to the search key words (COVID_19 Vaccine design and 
safety). The remaining were 94 article and after exclusion of doubled papers (25) there were 69. Unfortunately, only 5 articles out of 69 are about safety of covid_19 vaccines. Out of these 5, only 1 article is designed as double blind randomized clinical trial to evaluate safety and efficacy of vaccine manufactured by Sinovac structured as summary of study protocol for a randomized control study ${ }^{4}$ and one article designed as interim analysis of 2 randomized clinical trials to assess inactivated Vaccine against SARS-COV-2 on safety and immunogenicity outcomes. ${ }^{5}$ The Sinovic ${ }^{4}$ protocol stated at main outcome to assess primary safety endpoint solicited and unsolicited local and systemic adverse reactions in a period of one week after vaccination of each age group, in adults aged from 18-59 years old and elders aged 60 years or older. Adverse reactions defined in the protocol as adverse events that have a reasonable causal relationship to vaccination.

The Interim analysis ${ }^{5}$ of ongoing randomized, double-blind, placebo-controlled, phase 1 and 2 clinical trials to assess an inactivated COVID-19 vaccine were conducted in Henan Province, China, among 96 healthy adults in phase 1, and 224 in phase 2 , they aged $18-59$ years. Study enrollment started on April 12, 2020. The analysis was conducted on June16, 2020, and updated on July 27, 2020. The study main outcomes and measures were in two weeks; the primary safety as combined adverse reactions 7 days after each injection, and the primary immunogenicity outcome was neutralizing antibody response 14 days after the whole-course of vaccination, measured by a $50 \%$ plaque reduction neutralization test against live severe acute respiratory syndrome coronavirus 2 (SARS-CoV-2). Study Concluded that patients had a low rate of adverse reactions and demonstrated immunogenicity; the study is ongoing to ensure the efficacy and longer-term adverse event assessment need phase 3 trials. $^{5}$

The remaining three articles are about prospects of safe COVID_19, learning from the past, development of safe and effective Covid_19 Vaccines and assessment of risk of disease enhancement with COVID_19 Vaccine. The three articles are published in the following journals subsequently: Science transitional medicine, Nature review microbiology and Vaccine.

The article published by Shan Su et al. ${ }^{6}$ focuses on review of Mechanisms of vaccine-associated disease enhancement. It gives learning lessons from the past regarding possible Vaccine- associated disease enhancement (VADE) and how it partially overlaps with antibody- dependent enhancement (ADE) and enhanced respiratory disease (ERD). In Contrary ERD, VADE involves only the vaccine- associated situation, furthermore it is not limited to respiratory disease. For example, serotype heterotypic infection by dengue virus sometimes cause severer hemorrhagic dengue fever in vaccinated individuals. This phenomenon mostly related to VADE, but not related to ERD. Vaccine- associated disease enhancement could be related to antibody- dependent furthermore type $2 \mathrm{t}$ helper cell dependent mechanisms. The article published by Barton et al. ${ }^{7}$ about the prospects of safe COVID_19 Vaccine, although it didn't report concern in phase 1 about VADE for COVID-19 vaccines up to time of publication on November $4^{\text {th }}, 2020$, it is relaying on future results could come from phase 3 efficacy trials with long-term follow-up. Phase 3 clinical trials outcome are waited till now to provide the most comprehensive data on the safety of COVID-19 vaccines and the potential risks of Vaccineassociated enhancement of disease (VAED) ${ }^{7}$

The third study considering the experts' opinions released Consensus summary report ${ }^{8}$ comes from meeting hold in March 2020 and has been published in the following May. Experts gave advices regarding disease enhancement in animal models, importance of continuous monitor of (VAED) during clinical trials and direct message to Vaccine developers, they have to seek individual and solid scientific advices from regulatory authorities. ${ }^{8}$

No other data have been released in scientific publications from Vaccine developers or regulatory authorities in both Scopus and Clarivate data bases about the details of efficacy, and longevity or safety profiles of the developing / developed vaccine based on 2 or 3 months or more clinical trial follow up. That means UpToDate the scientific community don't have any published Randomized clinical trials with appropriate sample size significant outcome findings, with proper enough follow up time. Even there are no data available for meta-analysis to give safety evidence about the long time use of the newly emerged COVID_19 Vaccines, neither (couple of years) nor couple of months.

Fig. 1 shows meta-analysis Filtration and article Selection and inclusion in the study.

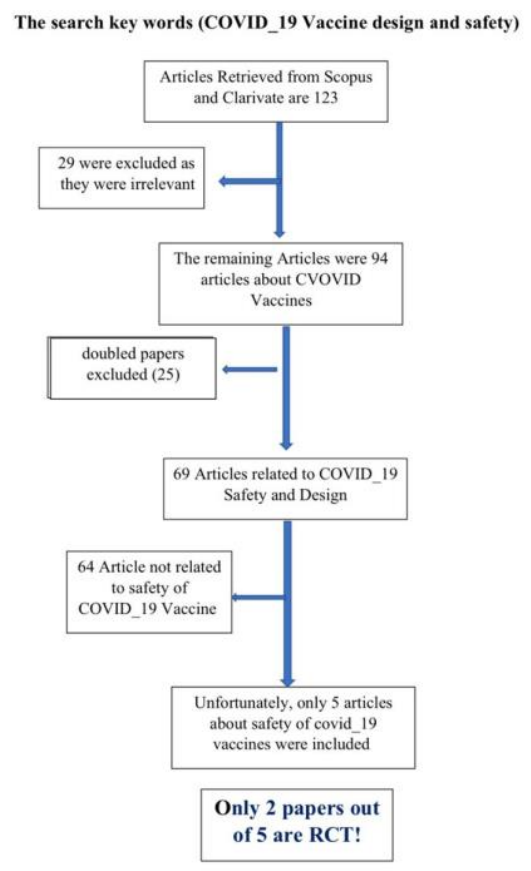

Figure 1: shows meta-analysis steps of articles' selection for inclusion in the study. 


\section{Vaccines' designs}

Many types of Vaccines' design and productions ${ }^{9,10}$ are now developing with different mechanisms of action. It well known that Viral Vector Vaccine are using harmless virus to carry the Corona virus gene inside it while the attenuated Vaccine is made of alive virus, but it is very weak, so stimulates immune system response without disease developing. Inactivated Vaccine is Vaccine made from killed or chemically, thermally or radiated viral particles, so Virus Can't replicate but provoke body immune response.

Interestingly, now many vaccines with different designs are under investigations in many countries. ${ }^{1,2,9}$ For example DNA Vaccine under developing / developed by Inovio Pharma, mRNA by Moderna and Pfizer/BioNtech, and Vector Vaccines by Oxford/AstraZenca, Gamalaya-Suptnik and the Janssen Vaccines. Lastly the example of live attenuated vaccine is one made by Griffith University, Codagenix and Indian Immunological Ltd while the Vaccine developed by Sinovac is an example for Inactivated Vaccine.

Possibly, another raised question is emerged regards the newly evolved mRNA and DNA Vaccines versus the traditional way of attenuated or inactivated Vaccines. Evidently the mechanism of induction of body immune response in mRNA and DNA Vaccines $^{2,9,10}$ is depending on engineered part of COVID virus gene enters the human cells protected by lipid Nanoparticle (LNP) or Plasmoid respectively to protect the genetic part degradation before it reaches the cell. Then the human cell transcribes and translates the genetic code into antigen (Mainly protein spike of Corona Virus) and display this antigen on the cell surface to stimulate an immune response. The Nanoparticles are manufactured from Aluminum hydroxide nano polymer, 9,11 and there is no single published article describing Vaccine safety regards human blood brain barrier or possible genetic errors during transcribing the viral part carrying the genetic code.

Based on the scanty available data in Scopus and Clarivate data bases it seems that the wide scale safety studies will not be achieved before administrations of varies vaccines for populations upon emergency grants from FDA and local health authorities in each country. This wide administration of vaccines could be a real part of the third phase clinical trial, but the world has no another choice.

Lastly an interesting article ${ }^{12}$ published in nature about how fast the new technology of mRNA by Moderna and BioNTech and DNA viral Vector by AstraZeneka wih Oxford UN. The article ${ }^{12}$ attributed the fast developing of COVID_19 Vaccine due to previous years of research in new technology and the supercharged fund. However, there is no sufficient details about that claimed years of research in new technology and results of previous Vaccines developed by the same technology according to our knowledge! Of course, and after data release, Author of this article totally agrees with Phillip B. ${ }^{12}$ in that the speedy technology approach used to develop SARS-CoV-2 Vaccine could really change the future of vaccine science. It could make the Vaccine developers more ready to react for further Virus Variants or Strains to protect against disease severity. ${ }^{13}$

Actually, how is the change in Vaccines future and How it would be the Humanity future? No scientist can answer. UpToDate No Scientific Clinical Evidence for long term Safety of Newly Evolved COVID_19 Vaccines: the world has to Know

In conclusion: Up to date there is a severe deficiency in scientific data released in scientific journals from all vaccine's developers regarding $3^{\text {rd }}$ phase clinical trials of COVID_19 vaccines regardless the new Covid_19 variants. Challenges for vaccine development exist during a pandemic, as well as concerns about safety on long terms. Evidence of safety could come any day and scientific community is waiting for hopeful findings after data release and completion of $3^{\text {rd }}$ phase clinical trials.

\section{REFERENCES}

1 - Graham, B. S. Rapid COVID-19 vaccine development. Science. 2020; 68: 945-946.

2- Thanh, Le, T. et al. The COVID-19 vaccine development landscape. Nat. Rev. Drug. Discov. 2020; 19: 305-306.

3- Shibo Jiang. Don't rush to deploy COVID-19 vaccines and drugs without sufficient safety guarantees. Nature 2020; 579: 321

4- Palacios, R., Patiño, E.G., de Oliveira Piorelli, R. et al. Double-Blind, Randomized, PlaceboControlled Phase III Clinical Trial to Evaluate the Efficacy and Safety of treating Healthcare Professionals with the Adsorbed COVID-19 (Inactivated) Vaccine Manufactured by Sinovac - PROFISCOV: A structured summary of a study protocol for a randomised controlled trial. Trials 2020; 21: 853.

5- Shengli Xia, Kai Duan, Yuntao Zhang, Dongyang Zhao, and Huajun Zhang, Effect of an Inactivated Vaccine Against SARS-CoV-2 on Safety and Immunogenicity Outcomes Interim Analysis of 2 Randomized Clinical 
Trials et al. JAMA. 2020;324(10):951-960. doi:10.1001/jama.2020.15543

6- Su, S., Du, L. \& Jiang, S. Learning from the past: development of safe and effective COVID19 vaccines. Nat Rev Microbiol.2020. https://doi.org/10.1038/s41579-020-00462-y

7- Barton F. Haynes, Lawrence Corey, Prabhavathi Fernandes, Peter B. Gilbert, Peter J. Hotez5, et al. Prospects for a safe COVID-19 vaccine. Sci. Transl. Med. 2020; 12, eabe0948 4 November 2020

8- Paul-Henri Lambert, Donna M. Ambrosino, Svein R. Andersen, Ralph S. Baric, Steven B. Black, et al. Consensus summary report for CEPI/BC March 12-13, 2020 meeting. Vaccine. 2020; (38) 31: 4783-4791.

9- Xingwu Zhou, Xing Jiang, Moyuan Qu, George E. Aninwene, II, Vadim Jucaud, James J. etal Moon, Engineering Antiviral Vaccines. ACS Nano $\quad 2020 ; \quad 14: \quad 12370-12389$. https://dx.doi.org/10.1021/acsnano.0c06109.

10-Martín Calvelo a, Ángel Piñeiro b, Rebeca Garcia-Fandino. An immersive journey to the molecular structure of SARS-CoV-2: Virtual reality in COVID-19. Computational and Structural Biotechnology Journal 2020; 18: 2621-2628

11-Hornung, V.; Bauernfeind, F.; Halle, A.; Samstad, E. O.; Kono, H.; Rock, K. L.; Fitzgerald, K. A.; Latz, E. Silica Crystals and Aluminum Salts Activate the NALP3 Inflammasome through. Phagosomal Destabilization. Nat. Immunol. 2008; 9: 847-856.

12-Phillip Ball The lightning-fast quest for COVID vaccines - and what it means for other diseases. The speedy approach used to tackle SARS-CoV-2 could change the future of vaccine science. Nature 2021; 589: 16-18

13-Essmat, Ahmed. Neurological Manifestations in Egyptian Covid-19 Patients. AIMJ accepted December 2020 